\title{
Face Recognition Using HOG Feature Extraction and SVM Classifier
}

\author{
Swarnima Singh ${ }^{1}$, Durgesh Singh ${ }^{2}$, Vikash Yadav ${ }^{3}$ \\ ABES Engineering College, Ghaziabad, U.P, India ${ }^{1,3}$, sswarnima.singh@ gmail.com ${ }^{1}$, vikash.yadav@ abes.ac.in ${ }^{3}$ \\ Allahabad University, U.P, India, durgesh.singhau@gmail.com²
}

\begin{abstract}
The intelligent interaction between the human and the computer is of vital importance to reach the primary and contemporary goals of artificial intelligence. Digital imaging in life and in common society and in science and technology has become completely digital and this transformation of visual imagination into mathematical algorithms and numerical developments is significant for researchers to use it in everyday digitized world we live today. Numerous algorithms are developed on face recognition techniques particularly in the last two to three decades. The LBP and HOG feature extraction algorithms are preferable for real time applications. HOG feature extraction technique has been used in this paper for person detection using image analysis. The PCA an important feature method in Eigen faces method is today an important brainwave for almost all the face recognition algorithms unfolded into new and better programming methods.
\end{abstract}

Key words: Feature extraction, Local Binary Pattern, Eigen face

\section{INTRODUCTION}

In this paper an intelligent human face detection system is developed which resembles specific object-class detection. Facial recognition systems have also been used to develop robust security systems for many autonomous and independent services emerging its broad use from surveillance systems in banking securities to terrorism protection systems where identification of person could be carried out on the airport and border crossings. Various social networking sites use the surveillance system for giving security to their users like tagging and profile lock.

The major objective is to create a facial recognition system that can recognize any kind of image and constantly make improvements and changes autonomously. Another important task is to be time efficient and run the system in real time. By removing the noise from the images such as light in the image, facial hairs etc.
The first step involved in creating a facial recognition through deep learning involves the comparison between two images that are alike are in their 128-dimension values are accepted by the system or network and other dissimilar picture is discarded.

The picture is then changed over to RBG color ordering, the input image is analyzed and the faces are identified and their relative 128-D encoding is computed. After training the network with the above mentioned work we can later perform classification by using $\mathrm{kNN}$ to make conclusive face classification. A match is made where each faces are compared using present encoding data set. The function returns a "TRUE/FALSE" value for each of the query [1].

\section{LITERATURE SURVEY}

Navneet Dalal et. al. [2] made a change in the outlook by introducing the HOG or the Histogram of oriented instead of Eigen faces which are the standard of PCA algorithms. The edge orientations are counted for HOG extraction in a local neighborhood of an image. They being densely overlapping in grid provide more accurate person detection. This is a programmed framework which restricts the facial highlights. The creator considered 68 facial focuses descriptors dependent on HOG which are invariable to enlightenment and pivot and has been applied in object acknowledgment and walker acknowledgment [5].

In early 1960s the researchers like Chan and Bisson worked on the human facial systems but the work was not much recognized back then. Few works were a part of development in late 1990s by few students of Bochum and university of California. Later in 2006 through United States Army Research Laboratory and 10 times better performance was observed in the algorithms. These algorithms were derived by distinctive landmarks on face like contour of eye sockets, nose, lips and chin. Relative newer technologies used the facial expressions like frowning, blinking, smiling.

The open $\mathrm{CV}$ face recognizer uses the Local Binary Pattern (LBP) [4] for texturing the image the by 
thresholding the neighborhood of every pixel and give binary number as resultant. The four parameters of LBPH [3] are radius, neighbor, grid $X$ and grid $Y$. Each image in the training set must have an ID. Firstly the image is enhanced using the sliding window on the basis of parameters like radius and neighbors.

A grayscale image is of window $3 \times 3$ pixel matrix with central threshold value. This value will elucidate the neighboring values of the 8 neighbors and given for each value of threshold a 0 is assigned to all values whose value is less than Threshold and value 1 for higher. The matrix will contain only 0 s and $1 \mathrm{~s}$ and hen this is converted to a decimal value. Using this image the histogram is extracted by Grid X and Grid Y parameters. This is done by splitting the image into multiple grids.

Histograms are compared using Euclidean distance, chi-square, and absolute value. The calculated distance is called confidence. Lower the confidence closer the histograms.

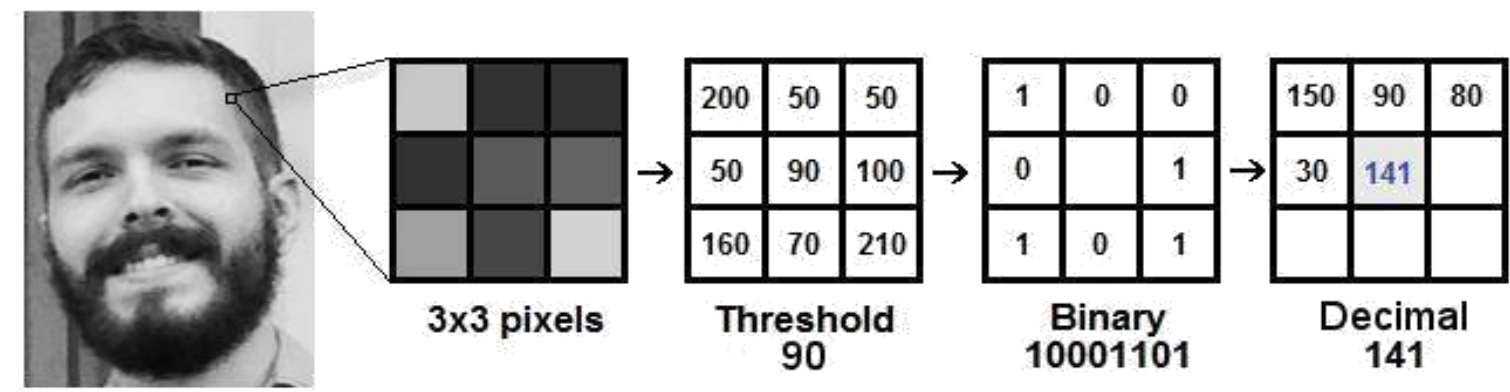

Figure 1: Various conversions of an image from pixels to decimal

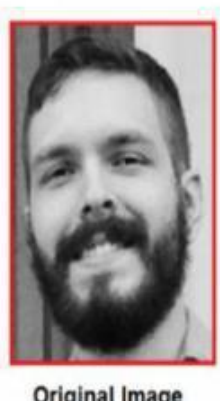

Original Image

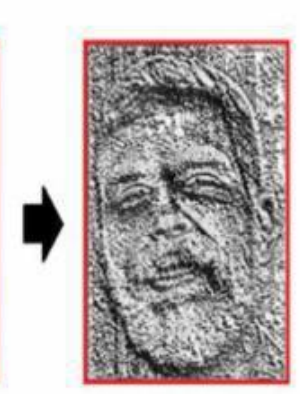

LBP Result

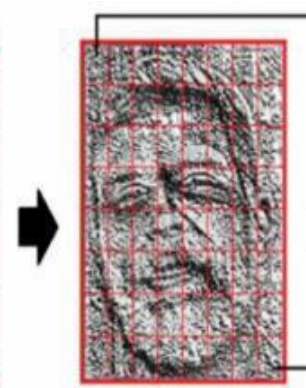

Regions/Grids (Grid X-Grid Y)

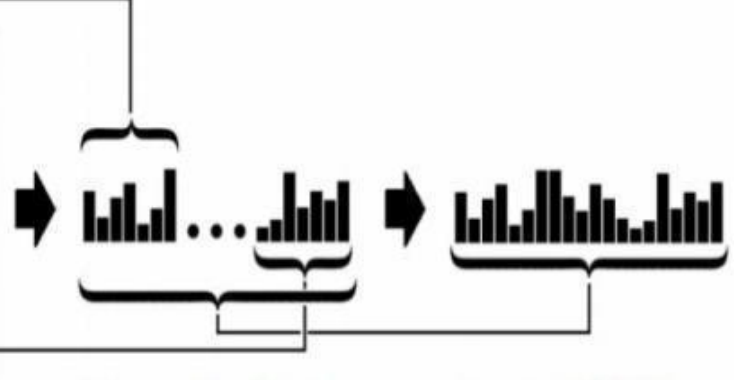

Histogram of each region

Concatenated Histogram

Figure 2: Concatenated and individual histograms of the original image

\section{IMPLEMENTATION}

The main steps consist of face detection, feature Extraction, data comparison and finally the face recognition ex "Hey this is Amanda (name)". The landmarks of the face are detected by Dlib and OpenCV. Through Dlib's state-of-the-art face recognition methodology built with deep learning technique 68 specific points could be applied on any of the following ranging from top of chin, the outside edge of eye etc. as shown in figure 3 . Here only basic transformations are used like rotation and scaling that preserves parallel lines called affine transformations.

Figure 4 shows the complete operation in which the face is detected and the landmarks are detected and transformations to get perfectly centered image. The encoding of the image is done where numerous images of a single person are taken and facial landmarks are extracted. A total of 2278 distance is calculated for 68 particular points. For recognition the image with minimum distance is in which there is maximum

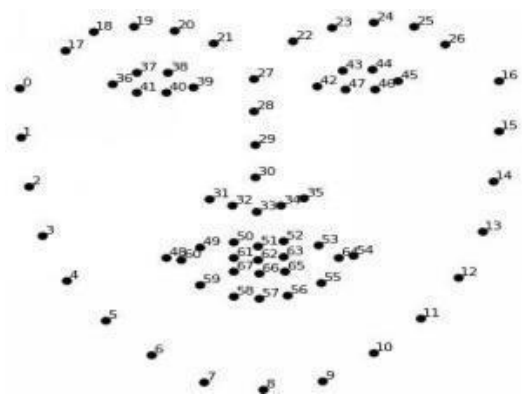

Figure 3: Depiction of 68 facial facial points

resemblance of the same person is considered the true image as output. To differentiate the unknowns there is an upper limit which is set and if difference is below that value, minimum value set represents the person.3.1 HOG Feature Vector Descriptor A feature vector converts an image of area distributed in three channels i.e. length, breadth and height of the picture to a feature vector of array of length $n$. 
Swarnima Singh et al., International Journal of Emerging Trends in Engineering Research, 8(9), September 2020, $6437-6440$

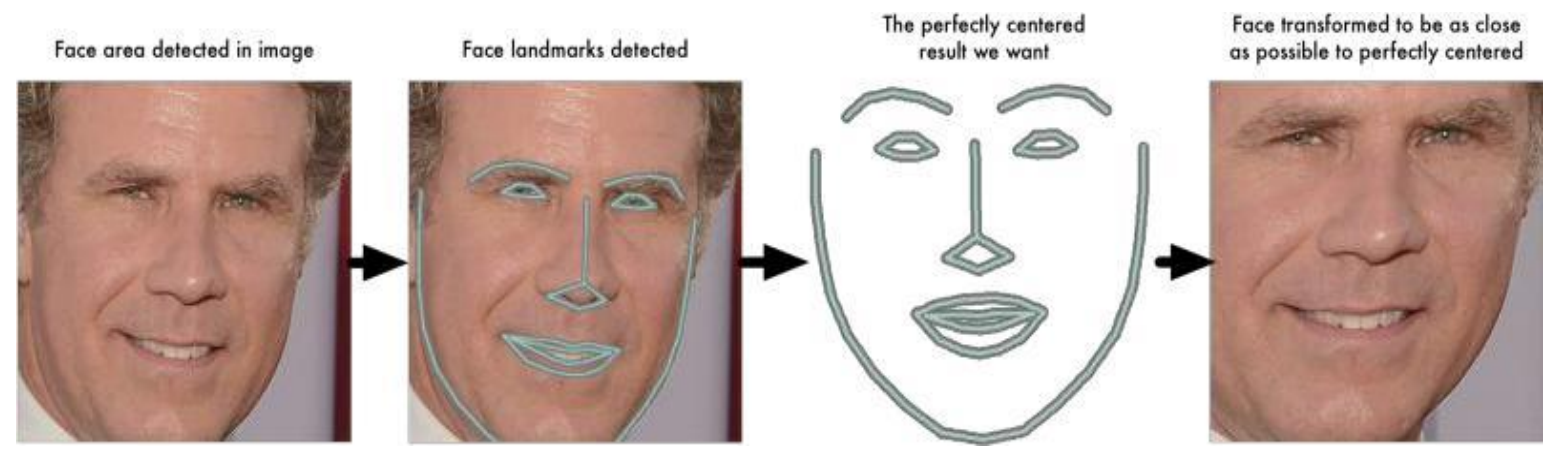

Figure 4: Facial landmarks extraction

In this case the input image is of size $64 \times 128 \times 3$ and output feature vector is of length 3780 . The gradient horizontally and vertically could be calculated as $\mathrm{gx}(\mathrm{x}$ gradient $)=\operatorname{df}(\mathrm{x}, \mathrm{y}) / \mathrm{dx}$ and $\operatorname{gy}(\mathrm{y}$ gradient $)=\mathrm{df}(\mathrm{x}, \mathrm{y}) / \mathrm{dy}$, where $\mathrm{g}$ is the magnitude and $\theta$ is the orientation of gradient. The picture is splitted into 8x8 unit cells and gradient is calculated for every cell. An $8 \times 8$ image patch contains $8 \times 8 \times 3=192$ pixels the gradient of this patch contains 2 values (magnitude and direction) which adds up-to $8 \times 8 \times 2=128$ values. Representation here becomes more compact and less prone to noise. HOG was used for a hiker detection. Initially $8 \times 8$ cells in a photo of a walker scaled $64 \times 128$ was suitable to detect active features like face, top of head etc.
The center image with arrows shows the gradient's direction and length shows the magnitude. Rightmost image show the numerals representation of the gradients in these $8 \times 8$ cells with one peripheral alteration of angles between 0 and 180 instead of 0 and 360 degree.

A histogram of gradients is created in these $8 \times 8$ cells. These cell consists of 9 bins correlative to angels $0,20,40 \ldots 160$. In figure 5 bin is selected based on the direction. An angle of 80 degree and magnitude of 2 is the corresponding value. So it adds 2 to the 5th bin. The red color circled pixel is of angle 10 degree and magnitude of 4 . The vote of pixel splits between the mid of pixels 0 and 20 bins.

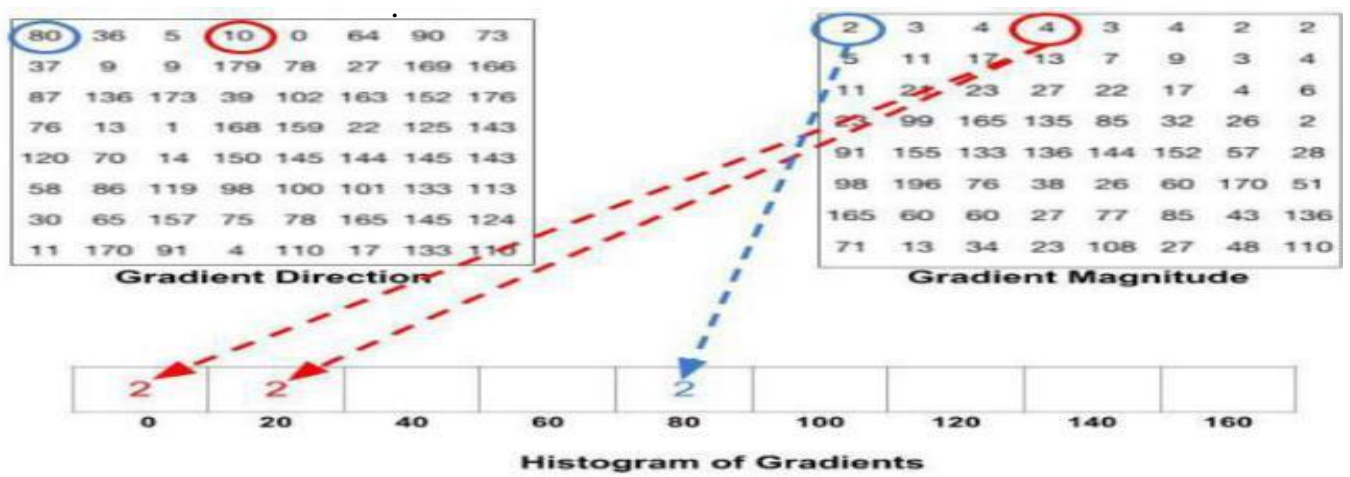

Figure 5: Bin selection
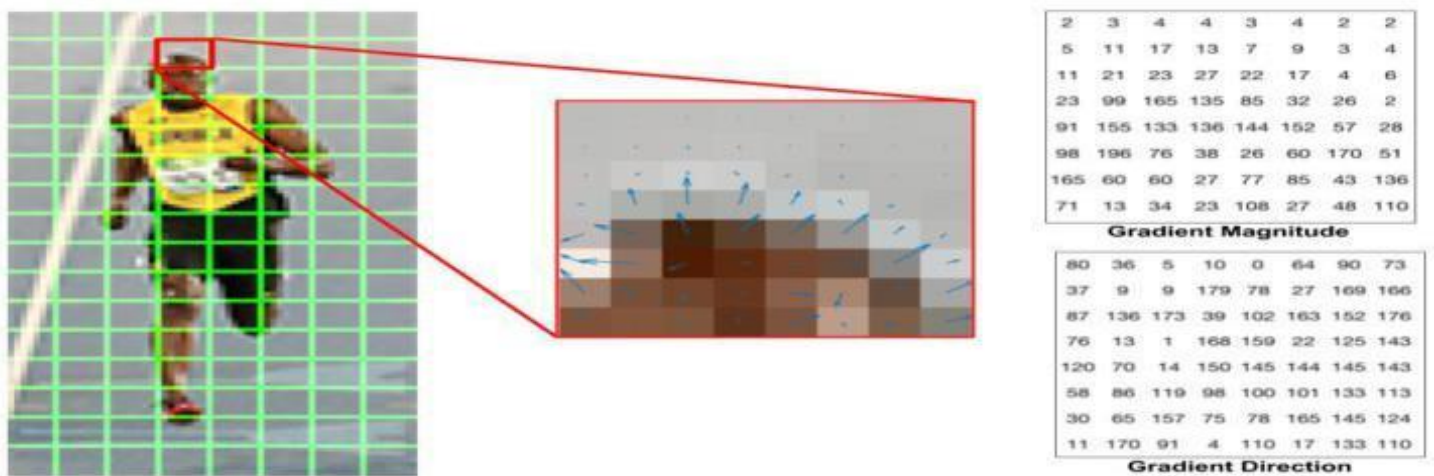

Figure 6: Hiker suitable gradient magnitude and direction [6] 


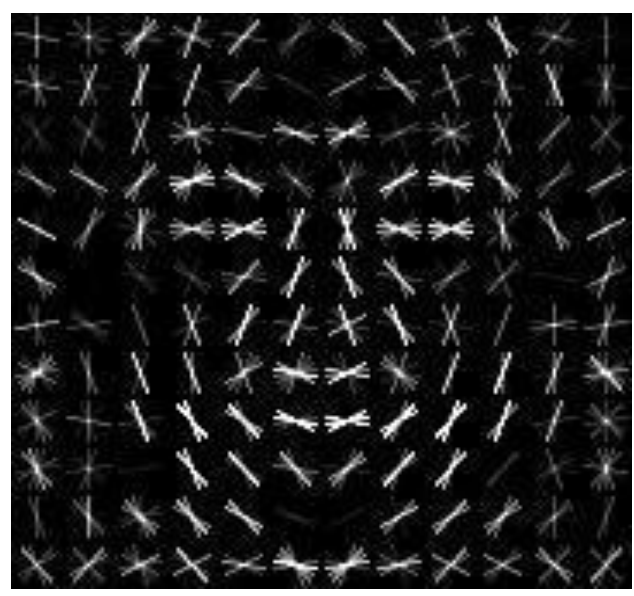

Figure 7: Trained Face

Figure represents a standard face that has been trained with 3000 images by Dlib library.

\section{CONCLUSION}

LBPH is one of the most challenging and helpful algorithms developed in the past few decades. It can represent local features in the images. It is almost impossible to detect and compare the imagery through video monitoring cameras. It is vigorous against gray scale transformations continuity. The LBPH algorithm gives the best performance in binary classification in OpenCV (Open Source Computer Vision Library) platform. The OpenCV LBPH algorithm has played a pivotal role the growing need for robust and sturdy security systems for identity in theft and crime investigations. Linear SVMs trained on HOG features have pervaded many visual recognition works in future.

\section{REFERENCES}

1. Zhang, W., Shan, S., Chen, X., \& Gao,W. (2017). "Local gabor binary patterns based on mutual information for face recognition." International Journal of Image and Graphics, 7(04), 777-793.

2. N,Dalal,B .Triggs, "Histogram of oriented gradients for Human Detection". 2005 IEEE computer society conference on computer vision and pattern recognition.

3. "LBPHOpenCV"https"//doc.opencv.org/2.4/modules/co ntrib/doc/facerec/facerectutorial.html\#local-bianry-patte rns-histograms".

4. Ahonen T Hadid A and Pietkainen, M (2006)."Face Description with local binary pattern: application to face Recognition". IEEE transactions on pattern analysis and machine intelligence.

5. Turk, M \& Pentland A. (1991) "Eigenfaces for Recognition". Journal of cognitive neural science.71- 86.
6. Hailing Zhou, Ajmal Mian, Lei Wei, Doug Creighton, Mo Hossny, and Saeid Nahavandi (December 2014). Recent Advances on Single Modal and Multimodal Face Recognition: A Survey. IEEE Transactions on Human-Machine System, 44(6), pp. 701-716. 